\title{
EQUISUMMABILITY OF CERTAIN SEQUENCES OF HADAMARD PRODUCTS OF TAYLOR SECTIONS AND INTERPOLATORY POLYNOMIALS
}

\author{
R. Brück, J. Müller, and A. Sharma
}

\begin{abstract}
In $[1,2]$ the classical equiconvergence theorem of Walsh was extended by the application of summability methods in order to enlarge the disk of equiconvergence to regions of equisummability. A further generalization was achieved in [3], where sequences of Hadamard products of a fixed power series with interpolatory polynomials were considered. The aim of this paper is to continue this work by investigating commutators of interpolatory polynomials and Hermite interpolatory polynomials.
\end{abstract}

\section{Introduction}

Let $g$ be a function holomorphic in the disk $\mathbb{D}_{R}:=\{z \in \mathbb{C}:|z|<R\}$ for some $R>1$, let $L_{n}(\cdot ; g)$ be the Lagrange interpolatory polynomial to $g$ in the $(n+1)$-st roots of unity, and denote by $S_{n}^{g}$ the $n$-th partial sum of the power series expansion of $g$ about 0 . Then the classical equiconvergence theorem of J. L. Walsh [8, p. 153] states that

$$
\lim _{n \rightarrow \infty}\left[L_{n}(z ; g)-S_{n}^{g}(z)\right]=0
$$

compactly in $\mathbb{D}_{R^{2}}$ (i.e., uniformly on compact subsets of $\mathbb{D}_{R^{2}}$ ).

In $[1,2]$ the first author applied certain summability methods in order to enlarge the disk of equiconvergence to regions of equisummability. Roughly speaking, one of his results may be stated as follows. If $A$ is a summability method which sums the geometric series $\gamma(z)=\sum_{\nu=0}^{\infty} z^{\nu}$ to $1 /(1-z)$ compactly in an open set $S$ containing the unit disk $\mathbb{D}$, then the sequence $\left(L_{n}(\cdot ; g)-S_{n}^{g}\right)$ is compactly $A$-summable to 0 in a certain open set $\mathcal{E}$. This set $\mathcal{E}$ always contains the disk $\mathbb{D}_{R^{2}}$, and it depends on $S$ and the singularities of $g$.

Recently, the first two authors [3] generalized this result by replacing the "test function" $\gamma$ by an arbitrary power series $f$, and by considering the sequence $\left(S_{n}^{f} *\left(L_{n}(\cdot ; g)-S_{n}^{g}\right)\right)$, where * denotes the Hadamard product of two power series.

The aim of this paper is to extend their result in two directions. In [5], Lou considered commutators of interpolatory polynomials, namely

$$
D_{m n}^{g}(z ; \alpha, \beta):=L_{m}\left(z ; \beta, L_{n}(\cdot ; \alpha, g)\right)-L_{n}\left(z ; \alpha, L_{m}(\cdot ; \beta, g)\right)
$$

Received August 23, 1993, revised February 16, 1994.

1991 Mathematics Subject Classification: 30E10, 40A30.

Key words and phrases: Walsh equiconvergence, equisummability, Hadamard product, Taylor sections, interpolatory polynomials.

The first author was supported by NSERC A3094, and wishes to express his thanks to Professor A. Sharma for his warm hospitality during a visit at the University of Alberta in May and June 1992. 
where $m=q(n+1)-1$ for some $q \in \mathbb{N}, \alpha, \beta \in \mathbb{D}_{R}$, and $L_{n}(\cdot ; \alpha, g)$ denotes the Lagrange interpolatory polynomial of $g$ in the roots of the equation $w^{n+1}-\alpha^{n+1}=0$, i.e., $L_{n}(w ; \alpha, g)=g(w)$, if $\alpha \neq 0$, and $L_{n}(z ; 0, g)=S_{n}^{g}(z)(z \in \mathbb{C})$. We will generalize Lou's result by applying summability methods $A$ to the sequence $\left(S_{n}^{f} * D_{m n}^{g}(\cdot ; \alpha, \beta)\right)$. Furthermore, we will generalize a theorem of Cavaretta, Sharma, and Varga [4] concerning Hermite interpolation.

This paper will be arranged as follows. In the first part, we give some notations and preliminaries concerning Hadamard products and summability methods. In the second part, we state our main results concerning commutators of interpolatory polynomials as considered by Lou [5], and Hermite interpolatory polynomials. The third part contains the proofs of the main results, and in the fourth part, we make some remarks on the existence of summability methods.

\section{Notations and preliminaries}

1.1. Hadamard product and its properties. For two power series $F(z)=$ $\sum_{\nu=0}^{\infty} a_{\nu} z^{\nu}$ and $G(z)=\sum_{\nu=0}^{\infty} b_{\nu} z^{\nu}$ with positive radii of convergence $R_{F}$ and $R_{G}$, respectively, the Hadamard product $F * G$ of $F$ and $G$ is defined by

$$
(F * G)(z):=\sum_{\nu=0}^{\infty} a_{\nu} b_{\nu} z^{\nu} .
$$

Obviously, the radius of convergence of $F * G$ is at least $R_{F} R_{G}$.

Furthermore, we need the Hadamard multiplication theorem in the version of the second author [6] which gives a lower estimate for the region of holomorphy of $F * G$. To state his result, we introduce the following notations. For arbitrary sets $A, B \subset \mathbb{C}$, $\alpha \in \mathbb{C}$ and $k \in \mathbb{N}$, we set $A^{c}:=\mathbb{C} \backslash A, A \cdot B:=\{a b: a \in A, b \in B\}, \alpha A:=\{\alpha\} \cdot A$, $A^{k}:=\left\{a^{k}: a \in A\right\}$, and $A * B:=\left(A^{c} \cdot B^{c}\right)^{c}$. If $0 \in A \cap B$, then it is easily seen that $0 \in A * B$, and

$$
A * B=\bigcap_{a \notin A} a B=\bigcap_{b \notin B} b A .
$$

For open sets $A, B \subset \mathbb{C}$ with $0 \in A \cap B$, the set $A * B$ is also open but not necessarily connected, even if $A$ and $B$ are so. If $0 \in A$, we denote by $A_{0}$ the component of $A$ which contains 0 . For an open set $\Omega \subset \mathbb{C}$, let $H(\Omega)$ be the topological vector space of all functions holomorphic in $\Omega$ with the usual topology of locally uniform convergence. Let $\varphi \in H(\Omega)$ for some open set $\Omega \subset \mathbb{C}$ such that $0 \in \Omega$. Then, for $a \in \mathbb{C}_{\infty} \backslash\{0\}$ (where $\mathbb{C}_{\infty}:=\mathbb{C} \cup\{\infty\}$ ) and $k \in \mathbb{N}_{0}:=\mathbb{N} \cup\{0\}$, we define $\varphi_{a, k} \in H(a \Omega$ ) (where $\infty \Omega=\mathbb{C})$ by

$$
\varphi_{a, k}(z):= \begin{cases}\left.\frac{1}{k !} \frac{d^{k}}{d u^{k}}\left(u^{k} \varphi(u)\right)\right|_{u=z / a} & \text { for } a \in \mathbb{C} \\ \frac{1}{k !} \varphi^{(k)}(0) z^{k} & \text { for } a=\infty\end{cases}
$$

Finally, we denote by $\gamma(z)$ the geometric series $\sum_{\nu=0}^{\infty} z^{\nu}$ as well as its analytic continuation $1 /(1-z)$.

Theorem $\mathbf{H}([6])$. Let $\Omega_{1}, \Omega_{2} \subset \mathbb{C}$ be open sets such that $0 \in \Omega_{1} \cap \Omega_{2}$. Then, for any $F \in H\left(\Omega_{1}\right)$, there exists a unique continuous linear map $T_{F}: H\left(\Omega_{2}\right) \rightarrow H\left(\Omega_{1} * \Omega_{2}\right)$ such that $T_{F}\left(\gamma_{a, k}\right)(z)=F_{a, k}(z)$ for all $z \in \Omega_{1} * \Omega_{2}, a \in \mathbb{C}_{\infty} \backslash \Omega_{2}$, and $k \in \mathbb{N}_{0}$. In particular, $T_{F}(G)=F * G$ in a neighbourhood of 0 for all $G \in H\left(\Omega_{2}\right)$, and therefore the power series $F * G$ admits an analytic continuation into the region $\left(\Omega_{1} * \Omega_{2}\right)_{0}$. 
In the following we always write $F * G$ instead of $T_{F}(G)$.

1.2. Summability methods. Let $X \subset \mathbb{R}$, and let $x^{*} \in \mathbb{R} \cup\{ \pm \infty\}$ be an accumulation point of $X$. Suppose further that $A=\left(a_{n}\right)_{n=0}^{\infty}$ is a sequence of complex-valued functions on $X$. A sequence $\left(s_{n}\right)_{n=0}^{\infty}$ of complex-valued functions defined in an open set $\Omega \subset \mathbb{C}$ is called compactly $A$-summable in $\Omega$ to the function $s$, if the series

$$
\sigma(x ; z):=\sum_{n=0}^{\infty} a_{n}(x) s_{n}(z)
$$

converges compactly in $\Omega$ (i.e., uniformly on compact subsets of $\Omega$ ) for all $x \in X$, and if

$$
\lim _{x \rightarrow x^{*}} \sigma(x ; z)=s(z)
$$

compactly in $\Omega$. In this case we write

$$
A-\lim _{n \rightarrow \infty} s_{n}(z)=s(z) \quad \text { compactly in } \Omega .
$$

Now we introduce three classes of summability methods $A$. For that purpose, let $f(z)=\sum_{\nu=0}^{\infty} f_{\nu} z^{\nu}$ be an arbitrary power series with positive radius of convergence $R_{f}$.

$(S)$ We say that $A$ satisfies the condition $(S)$ for some open set $S \subset \mathbb{C}$ containing 0 , if the power series

$$
\phi(x ; w):=\sum_{n=0}^{\infty} a_{n}(x) w^{n}
$$

converges for all $w \in \mathbb{C}$ and all $x \in X$, and if

$$
\lim _{x \rightarrow x^{*}} \phi(x ; w)=0 \quad \text { compactly for } w \in S .
$$

$(\Omega, f)$ We say that $A$ satisfies the condition $(\Omega, f)$ for some open set $\Omega \subset \mathbb{C}$ containing 0 and some power series $f$, if the power series in two variables

$$
\phi^{f}(x ; u, w):=\sum_{n=0}^{\infty} \sum_{\nu=0}^{\infty} a_{n+\nu}(x) f_{\nu} u^{\nu} w^{n}
$$

converges for all $(u, w) \in \mathbb{C} \times \mathbb{D}$, and all $x \in X$, and if

$$
\lim _{x \rightarrow x^{*}} \phi^{f}(x ; u, w)=0 \quad \text { compactly for }(u, w) \in \Omega \times \mathbb{D},
$$

where $\mathbb{D}:=\mathbb{D}_{1}$.

$(\Omega, f, p)$ We say that $A$ satisfies the condition $(\Omega, f, p)$ for some open set $\Omega \subset \mathbb{C}$ containing 0 , some power series $f$ and some $p \in \mathbb{N}$, if the series

$$
\phi_{j}^{f}(x ; u, w, v):=\sum_{n=0}^{\infty} \sum_{\nu=0}^{\infty} a_{n+\nu}(x) F_{\nu j}(v) u^{\nu} w^{n}
$$

converges for all $(u, w, v) \in \mathbb{C} \times \mathbb{D} \times \mathbb{C}$, all $x \in X$, and $j=1, \ldots, p$, where

$$
F_{\nu j}(v):=\sum_{s=0}^{j-1} f_{\nu j+s} v^{s} \quad\left(v \in \mathbb{C}, j \in \mathbb{N}, \nu \in \mathbb{N}_{0}\right),
$$


and if

$$
\lim _{x \rightarrow x^{*}} \phi_{j}^{f}(x ; u, w, v)=0 \quad \text { compactly for }(u, w, v) \in \Omega \times \mathbb{D} \times \mathbb{C}
$$

and for $j=1, \ldots, p$.

Note that the condition $(\Omega, f, 1)$ coincides with the condition $(\Omega, f)$. For the existence of such summability methods, we refer to Section 4 .

\section{Statement of results}

2.1. Commutators of interpolatory polynomials. Let $G \subset \mathbb{C}$ be a region containing the disk $\mathbb{D}_{R}:=\{z \in \mathbb{C}:|z|<R\}$ for some $R>1$, and let $g \in H(G)$. Furthermore, let

$$
f(z)=\sum_{\nu=0}^{\infty} f_{\nu} z^{\nu}
$$

be an arbitrary power series with positive radius of convergence $R_{f}$. For $q \in \mathbb{N}$, $n \in \mathbb{N}_{0}, m=q(n+1)-1$, and $\alpha, \beta \in \mathbb{D}_{R}$, we set

$$
D_{m n}^{f, g}(z ; \alpha, \beta):=\left(S_{n}^{f} * D_{m n}^{g}(\cdot ; \alpha, \beta)\right)(z) \quad(z \in \mathbb{C}),
$$

where $D_{m n}^{g}(z ; \alpha, \beta)$ is defined by $(0.1)$. Since $m \geq n$, we have

$$
D_{m n}^{g}(z ; \alpha, \beta)=L_{n}(z ; \alpha, g)-L_{n}\left(z ; \alpha, L_{m}(\cdot ; \beta, g)\right) \quad(z \in \mathbb{C}) .
$$

In [5], Lou proved that

$$
\lim _{n \rightarrow \infty} D_{m n}^{g}(z ; \alpha, \beta)=0 \quad \text { compactly in } \mathbb{D}_{\tilde{R}}
$$

where

$$
\tilde{R}:=\frac{R^{1+q}}{\max \left\{|\alpha|^{q},|\beta|^{q}\right\}} .
$$

We will generalize this result by applying summability methods $A$ of the form $(\Omega, f)$ to the sequence $\left(D_{m n}^{f, g}(\cdot, \alpha, \beta)\right)$. For that purpose, we define the sets

$$
\begin{gathered}
\mathcal{E}_{1}:=\mathcal{E}_{1}(G, \Omega):=\bigcap_{k=q}^{\infty} \bigcap_{c \notin G}\left(c\left(\frac{c}{\alpha}\right)^{k} \Omega\right), \\
\mathcal{E}_{2}:=\mathcal{E}_{2}(G, \Omega):=\bigcap_{k=0}^{q-1} \bigcap_{l=1}^{\infty} \bigcap_{c \notin G}\left(c\left(\frac{c}{\alpha}\right)^{k}\left(\frac{c}{\beta}\right)^{l q} \Omega\right),
\end{gathered}
$$

and

$$
\mathcal{E}:=\mathcal{E}(G, \Omega):=\mathcal{E}_{1} \cap \mathcal{E}_{2},
$$

where we set $c / 0:=\infty$ for $c \neq 0, \infty^{k}:=\infty$, if $k \in \mathbb{N}$, and $\infty^{k}:=1$, if $k=0$.

Theorem 2.1. If $A$ satisfies the condition $(\Omega, f)$ for some $\Omega$ and $f$, then there holds for every $g \in H(G)$

$$
A-\lim _{n \rightarrow \infty} D_{m n}^{f, g}(z ; \alpha, \beta)=0 \quad \text { compactly in } \mathcal{E}(G, \Omega)
$$


Remarks. (i) If $\mathbb{D}_{R^{\prime}} \subset \Omega$ for some $R^{\prime}>0$, then $\mathcal{E} \supset \mathbb{D}_{\tilde{R}}$, where

$$
\tilde{R}:=\frac{R^{\prime} R^{1+q}}{\max \left\{|\alpha|^{q},|\beta|^{q}\right\}}
$$

and $\mathcal{E}$ is an open set. In the special case $f=\gamma$ and $R^{\prime}=1$, Theorem 2.1 and Lemma 4.1 (see Section 4) imply Theorem 3.1 of [1] (in a slightly weaker version), which itself is a generalization of the above mentioned result of Lou [5]. Furthermore, for $\alpha=0, \beta=1$, and $q=1$, we obtain Theorem 1 of [3] which generalizes Theorem 1 of $[2]$.

(ii) If $\alpha=0$ and $\beta \neq 0$, then $\mathcal{E}_{1}=\mathbb{C}, \mathcal{E}_{2}=\bigcap_{l=1}^{\infty} \bigcap_{c \notin G}\left(c(c / \beta)^{l q} \Omega\right)$, and $D_{m n}^{f, g}(z ; 0, \beta)=$ $\left(S_{n}^{f} *\left(S_{n}^{g}-S_{n}^{L_{m}(\cdot ; \beta, g)}\right)\right)(z)$, so that $\mathcal{E} \supset \mathbb{D}_{R^{\prime} R^{1+q} /|\beta|^{q}}$. In particular, for $\beta=1, f=\gamma$, and $A$ being the usual convergence, Theorem 2.1 and Lemma 4.1 imply Theorem 1 of Rivlin [7].

(iii) If $\beta=0$ and $\alpha \neq 0$, then $\mathcal{E}_{2}=\mathbb{C}, \mathcal{E}_{1}=\bigcap_{k=q}^{\infty} \bigcap_{c \notin G}\left(c(c / \alpha)^{k} \Omega\right)$, and $D_{m n}^{f, g}(z ; \alpha, 0)=$ $\left(S_{n}^{f} *\left(L_{n}(\cdot ; \alpha, g)-L_{n}\left(\cdot ; \alpha, S_{m}^{g}\right)\right)\right)(z)$, so that $\mathcal{E} \supset \mathbb{D}_{R^{\prime} R^{1+q} /|\alpha|^{q}}$. In particular, for $\alpha=1, f=\gamma$, and $A$ being the usual convergence, Theorem 2.1 and Lemma 4.1 imply Theorem 1 of Cavaretta, Sharma, and Varga [4].

(iv) For the existence of summability methods $A$ satisfying the condition $(\Omega, f)$ we refer to Lemma 4.2.

Corollary 2.1. If $A$ satisfies the condition $(S)$ for some open set $S \supset \mathbb{D}$, and if $\Omega^{\prime} \subset \mathbb{C}$ is an open set containing 0 , then there holds for every $f \in H\left(\Omega^{\prime}\right)$ and every $g \in H(G)$,

$$
A-\lim _{n \rightarrow \infty} D_{m n}^{f, g}(z ; \alpha, \beta)=0 \quad \text { compactly in } \tilde{\mathcal{E}}:=\mathcal{E}\left(G, S, \Omega^{\prime}\right)
$$

where

$$
\tilde{\mathcal{E}}\left(G, S, \Omega^{\prime}\right):=\mathcal{E}\left(G, \Omega^{\prime} * S\right)=\Omega^{\prime} * \mathcal{E}(G, S)=\bigcap_{\omega \notin \Omega^{\prime}}(\omega \mathcal{E}(G, S)) .
$$

Proof. The assertion follows immediately by combining Lemma 4.2 (see Section 4) and Theorem 2.1.

Remark. If $\mathbb{D}_{R^{\prime}} \subset \Omega^{\prime}$ for some $R^{\prime}>0$, then $\tilde{\mathcal{E}} \supset \mathbb{D}_{\tilde{R}}$, where $\tilde{R}$ is defined by (2.1). Therefore, Corollary 2.1 also generalizes all results mentioned in the remarks after Theorem 2.1.

2.2. Hermite interpolation. For $p \in \mathbb{N}$ and $n \in \mathbb{N}_{0}$, we denote by $H_{p(n+1)-1}(\cdot ; g)$ the Hermite interpolatory polynomial of $g, g^{\prime}, \ldots, g^{(p-1)}$ in the $(n+1)$-st roots of unity, i.e.,

$$
H_{p(n+1)-1}^{(k)}(w ; g)=g^{(k)}(w) \quad(k=0,1, \ldots, p-1)
$$

for all $w \in \mathbb{C}$ satisfying $w^{n+1}=1$. Then we set

$$
D_{p n}^{g}(z):=H_{p(n+1)-1}(z ; g)-S_{p(n+1)-1}^{g}(z) \quad(z \in \mathbb{C})
$$

and

$$
D_{p n}^{f, g}(z):=\left(S_{p(n+1)-1}^{f} * D_{p n}^{g}\right)(z) \quad(z \in \mathbb{C}) .
$$

In [4] Cavaretta, Sharma, and Varga proved that

$$
\lim _{n \rightarrow \infty} D_{p n}^{g}(z)=0 \quad \text { compactly in } \mathbb{D}_{R^{1+1 / p}} .
$$


We will generalize this result by applying summability methods $A$ of the form $(\Omega, f, p)$ to the sequence $\left(D_{p n}^{f, g}\right)$. For that purpose, we define the set

$$
\mathcal{E}_{H}:=\mathcal{E}_{H}(G, \Omega):=\bigcap_{\substack{k=0 \\(k, j) \neq(0, p)}}^{\infty} \bigcap_{\substack{j=1 \\ c \notin G}} \bigcap_{j}^{-1}\left(c^{k+p} \Omega\right),
$$

where $\varphi_{j}: \mathbb{C} \rightarrow \mathbb{C}$ is defined by $\varphi_{j}(z):=z^{j}$.

Theorem 2.2. If $A$ satisfies the condition $(\Omega, f, p)$ for some $\Omega$ and $f$, then there holds for every $g \in H(G)$

$$
A-\lim _{n \rightarrow \infty} D_{p n}^{f, g}(z)=0 \quad \text { compactly in } \mathcal{E}_{H}(G, \Omega) .
$$

Remarks. (i) If $\mathbb{D}_{R^{\prime}} \subset \Omega$ for some $R^{\prime}>0$, then $\mathcal{E}_{H} \supset \mathbb{D}_{\tilde{R}}$, where

$$
\tilde{R}:= \begin{cases}R^{\prime} R^{2} & \text { for } p=1, \\ R\left(R^{\prime} R\right)^{1 / p} & \text { for } R^{\prime} \geq \frac{1}{R}, \quad p \geq 2 \\ \left(R^{\prime} R^{p}\right)^{1 /(p-1)} & \text { for } \frac{1}{R^{p}} \leq R^{\prime}<\frac{1}{R}, \quad p \geq 2, \\ R^{\prime} R^{p} & \text { for } R^{\prime}<\frac{1}{R^{p}}, \quad p \geq 2,\end{cases}
$$

and $\mathcal{E}_{H}$ is an open set. In the special case $f=\gamma$ and $R^{\prime}=1$, Theorem 2.2 and Lemma 4.3 (see Section 4) imply a special version of Theorem 4.1 of [1] which itself is a generalization of the above mentioned result of Cavaretta, Sharma, and Varga [4]. Furthermore, for $p=1$ we obtain Theorem 1 of [3].

(ii) For the existence of summability methods $A$ satisfying the condition $(\Omega, f, p)$, we refer to Lemma 4.3.

Corollary 2.2. If $A$ satisfies the condition $(S)$ for some open set $S \supset \mathbb{D}$, and if $\Omega^{\prime} \subset \mathbb{C}$ is an open set containing 0 , then there holds for every $f \in H\left(\Omega^{\prime}\right)$ and every $g \in H(G)$

$$
A-\lim _{n \rightarrow \infty} D_{p n}^{f, g}(z)=0 \quad \text { compactly in } \tilde{\mathcal{E}}_{H}:=\tilde{\mathcal{E}}_{H}\left(G, S, \Omega^{\prime}\right)
$$

where

$$
\tilde{\mathcal{E}}_{H}\left(G, S, \Omega^{\prime}\right):=\mathcal{E}_{H}\left(G, \Omega^{\prime} * S\right)=\Omega^{\prime} * \mathcal{E}_{H}(G, S)=\bigcap_{\omega \notin \Omega^{\prime}}\left(\omega \mathcal{E}_{H}(G, S)\right)
$$

Proof. The assertion follows immediately by combining Lemma 4.3 (see Section 4) and Theorem 2.2.

Remark. If $\mathbb{D}_{R^{\prime}} \subset \Omega^{\prime}$ for some $R^{\prime}>0$, then $\tilde{\mathcal{E}}_{H} \supset \mathbb{D}_{\tilde{R}}$, where $\tilde{R}:=R^{\prime} R^{1+1 / p}$. Therefore, Corollary 2.2 also generalizes all results mentioned in the remarks after Theorem 2.2. 


\section{Proofs of main theorems}

We recall the well-known interpolation formula of Hermite

$$
\begin{aligned}
L_{n}(z ; \alpha, g) & =\frac{1}{2 \pi i} \int_{|t|=r} \frac{g(t)}{t-z} \frac{t^{n+1}-z^{n+1}}{t^{n+1}-\alpha^{n+1}} d t \\
& =\frac{1}{2 \pi i} \int_{|t|=r} g(t) \frac{1}{t^{n+1}-\alpha^{n+1}} \sum_{\nu=0}^{n} t^{n-\nu} z^{\nu} d t \quad(z \in \mathbb{C}),
\end{aligned}
$$

where $|\alpha|<r<R$. Therefore,

$$
\begin{aligned}
\left(S_{n}^{f} * L_{n}(\cdot ; \alpha, g)\right)(z) & =\frac{1}{2 \pi i} \int_{|t|=r} g(t) \frac{1}{t^{n+1}-\alpha^{n+1}} \sum_{\nu=0}^{n} t^{n-\nu} f_{\nu} z^{\nu} d t \\
& =\frac{1}{2 \pi i} \int_{|t|=r} g(t) S_{n}^{f}\left(\frac{z}{t}\right) \frac{t^{n+1}}{t^{n+1}-\alpha^{n+1}} \frac{d t}{t} \quad(z \in \mathbb{C}) .
\end{aligned}
$$

In particular, we obtain for $\alpha=0$

$$
\left(S_{n}^{f} * S_{n}^{g}\right)(z)=\frac{1}{2 \pi i} \int_{|t|=r} g(t) S_{n}^{f}\left(\frac{z}{t}\right) \frac{d t}{t} \quad(z \in \mathbb{C}) .
$$

Proof of Theorem 2.1. We divide the proof into several steps.

STEP 1. At first we derive an integral representation for $D_{m n}^{f, g}(\cdot ; \alpha, \beta)$. For that purpose, let $\max \{|\alpha|,|\beta|\}<r<R$. Then we have by (3.1)

$$
\begin{aligned}
L_{m}(z ; \beta, g) & =\frac{1}{2 \pi i} \int_{|t|=r} \frac{g(t)}{t^{m+1}-\beta^{m+1}} \sum_{\mu=0}^{m} t^{m-\mu} z^{\mu} d t \\
& =\frac{1}{2 \pi i} \int_{|t|=r} \frac{g(t)}{t^{m+1}-\beta^{m+1}} \sum_{k=0}^{q-1} z^{k(n+1)} \sum_{\nu=0}^{n} t^{m-\nu-k(n+1)} z^{\nu} d t \quad(z \in \mathbb{C}),
\end{aligned}
$$

and therefore,

$$
\begin{aligned}
L_{n}\left(z ; \alpha, L_{m}(\cdot ; \beta, g)\right) & =\frac{1}{2 \pi i} \int_{|t|=r} \frac{g(t)}{t^{m+1}-\beta^{m+1}} \sum_{k=0}^{q-1} \alpha^{k(n+1)} \sum_{\nu=0}^{n} t^{m-\nu-k(n+1)} z^{\nu} d t \\
& =\frac{1}{2 \pi i} \int_{|t|=r} \frac{g(t)}{t-z} \frac{t^{m+1}-\alpha^{m+1}}{t^{m+1}-\beta^{m+1}} \frac{t^{n+1}-z^{n+1}}{t^{n+1}-\alpha^{n+1}} d t \quad(z \in \mathbb{C}) .
\end{aligned}
$$

Subtraction of (3.4) from (3.1) yields

$$
D_{m n}^{g}(z ; \alpha, \beta)=\frac{1}{2 \pi i} \int_{|t|=r} \frac{g(t)}{t-z} \frac{\alpha^{m+1}-\beta^{m+1}}{t^{m+1}-\beta^{m+1}} \frac{t^{n+1}-z^{n+1}}{t^{n+1}-\alpha^{n+1}} d t \quad(z \in \mathbb{C}) .
$$

Proceeding as in the proof of (3.2), we obtain

$$
D_{m n}^{f, g}(z ; \alpha, \beta)=\frac{1}{2 \pi i} \int_{|t|=r} g(t) S_{n}^{f}\left(\frac{z}{t}\right) K_{q, n}(t ; \alpha, \beta) \frac{d t}{t} \quad(z \in \mathbb{C}),
$$


where (note that $m=q(n+1)-1)$

$$
K_{q, n}(t ; \alpha, \beta):=\frac{t^{n+1}\left(\alpha^{m+1}-\beta^{m+1}\right)}{\left(t^{m+1}-\beta^{m+1}\right)\left(t^{n+1}-\alpha^{n+1}\right)} .
$$

STEP 2. Setting

$$
\psi(x ; z):=\sum_{n=0}^{\infty} a_{n}(x) D_{m n}^{f, g}(z ; \alpha, \beta)
$$

we see that $\psi(x ; \cdot)$ is an entire function for every $x \in X$ and

$$
\begin{aligned}
\psi(x ; z) & =\frac{1}{2 \pi i} \int_{|t|=r} g(t) \sum_{n=0}^{\infty} \sum_{\nu=0}^{n} a_{n}(x) f_{\nu}\left(\frac{z}{t}\right)^{\nu} K_{q, n}(t ; \alpha, \beta) \frac{d t}{t} \\
& =\frac{1}{2 \pi i} \int_{|t|=r} g(t) \sum_{\nu=0}^{\infty} \sum_{n=\nu}^{\infty} a_{n}(x) f_{\nu}\left(\frac{z}{t}\right)^{\nu} K_{q, n}(t ; \alpha, \beta) \frac{d t}{t} \\
& =\frac{1}{2 \pi i} \int_{|t|=r} g(t) \sum_{\nu=0}^{\infty} \sum_{n=0}^{\infty} a_{n+\nu}(x) f_{\nu}\left(\frac{z}{t}\right)^{\nu} K_{q, n+\nu}(t ; \alpha, \beta) \frac{d t}{t} \quad(z \in \mathbb{C}) .
\end{aligned}
$$

Now we write

$$
\begin{aligned}
K_{q, n}(t ; \alpha, \beta)= & \frac{1}{1-(\alpha / t)^{n+1}} \cdot \frac{(\alpha / t)^{m+1}-(\beta / t)^{m+1}}{1-(\beta / t)^{m+1}} \\
= & {\left[\left(\frac{\alpha}{t}\right)^{m+1}-\left(\frac{\beta}{t}\right)^{m+1}\right] \sum_{k=0}^{\infty}\left(\frac{\alpha}{t}\right)^{k(n+1)} \sum_{j=0}^{\infty}\left(\frac{\beta}{t}\right)^{j(m+1)} } \\
= & \sum_{k=0}^{\infty} \sum_{j=0}^{\infty}\left(\frac{\alpha}{t}\right)^{(k+q)(n+1)}\left(\frac{\beta}{t}\right)^{j q(n+1)} \\
& \quad-\sum_{k=0}^{\infty} \sum_{j=0}^{\infty}\left(\frac{\alpha}{t}\right)^{k(n+1)}\left(\frac{\beta}{t}\right)^{(j+1) q(n+1)}
\end{aligned}
$$

and we set, for $k, j \in \mathbb{N}_{0}$,

$$
T_{k j}(x ; z):=\frac{1}{2 \pi i} \int_{|t|=r} g(t)\left(\frac{\alpha}{t}\right)^{k}\left(\frac{\beta}{t}\right)^{j q} \phi^{f}\left(x ; \frac{z}{t}\left(\frac{\alpha}{t}\right)^{k}\left(\frac{\beta}{t}\right)^{j q},\left(\frac{\alpha}{t}\right)^{k}\left(\frac{\beta}{t}\right)^{j q}\right) \frac{d t}{t}
$$

where $\phi^{f}(x ; u, w)$ is given by (1.1). Putting (3.5), (3.6), and (3.7) together, we obtain

$$
\psi(x ; z)=\sum_{k=q}^{\infty} T_{k 0}(x ; z)-\sum_{k=0}^{q-1} \sum_{j=1}^{\infty} T_{k j}(x ; z)
$$


STEP 3. Now we consider a compact subset $C$ of $\mathcal{E}$. We have

$$
\left|\frac{\alpha}{t}\right|^{k} \leq \frac{|\alpha|}{r}<1 \text { for all } k \in \mathbb{N}
$$

and

$$
\left|\frac{\alpha}{t}\right|^{k}\left|\frac{\beta}{t}\right|^{j q} \leq \frac{|\beta|}{r}<1 \quad \text { for all } k \in \mathbb{N}_{0}, j \in \mathbb{N} .
$$

Let $\mu:=\max \{|z|: z \in C\}$, and choose $\delta>0$ such that $\overline{\mathbb{D}}_{\delta} \subset \Omega$. Then there exists $N \in \mathbb{N}$ sufficiently large such that $N \geq q$,

$$
\left|\frac{z}{t}\left(\frac{\alpha}{t}\right)^{k}\right| \leq \delta \quad \text { for }|t|=r,|z| \leq \mu, k \geq N
$$

and

$$
\left|\frac{z}{t}\left(\frac{\alpha}{t}\right)^{k}\left(\frac{\beta}{t}\right)^{j q}\right| \leq \delta \quad \text { for }|t|=r,|z| \leq \mu, k \in \mathbb{N}_{0}, j \geq N .
$$

Setting $M(r, g):=\max \{|g(t)|:|t|=r\}$ and

$$
\varepsilon(x):=\max \left\{\left|\phi^{f}(x ; u, w)\right|:|u| \leq \delta,|w| \leq \frac{1}{r} \max \{|\alpha|,|\beta|\}\right\},
$$

we obtain for $z \in C$

$$
\begin{aligned}
\mid \sum_{k=N}^{\infty} T_{k 0}(x ; z) & -\sum_{k=0}^{q-1} \sum_{j=N}^{\infty} T_{k j}(x ; z) \mid \\
\leq & \varepsilon(x) M(r, g)\left(\sum_{k=N}^{\infty}\left(\frac{|\alpha|}{r}\right)^{k}+\sum_{k=0}^{q-1} \sum_{j=N}^{\infty}\left(\frac{|\alpha|}{r}\right)^{k}\left(\frac{|\beta|}{r}\right)^{j q}\right) \\
\leq & \varepsilon(x) M(r, g) \frac{r}{r-|\alpha|} \frac{r^{q}}{r^{q}-|\beta|^{q}} \rightarrow 0 \quad\left(x \rightarrow x^{*}\right) .
\end{aligned}
$$

STEP 4. Finally, it suffices to consider a single term $T_{k j}(x ; z)$ for fixed $k, j \in$ $\{1, \ldots, N-1\}$. For simplicity, we assume that $\alpha \neq 0$ and $\beta \neq 0$, because the other cases can be handled similarly. We set

$$
E:=\left\{\zeta^{-1}: \zeta \notin \Omega\right\} \cup\{0\},
$$

and consider

$$
K:=\left\{\omega \in \mathbb{C}: \omega\left(\frac{\omega}{\alpha}\right)^{k}\left(\frac{\omega}{\beta}\right)^{j q} \in C \cdot E\right\} .
$$

Since $0 \in \Omega, E$ is a compact set, and therefore, it is easy to see that also $K$ is a compact set. We show that $K \subset G$. For that purpose let $c \notin G$. Then $z \in c(c / \alpha)^{k}(c / \beta)^{j q} \Omega$ for all $z \in C$, or $(z / c)(\alpha / c)^{k}(\beta / c)^{j q} \in \Omega$ for all $z \in C$. By the definition of $E$ this implies $(c / z)(c / \alpha)^{k}(c / \beta)^{j q} \notin E$ for all $z \in C$, or $c(c / \alpha)^{k}(c / \beta)^{j q} \notin C \cdot E$ which yields $c \notin K$. Therefore, we can choose a cycle $\Gamma$ such that

$$
\operatorname{ind}_{\Gamma}(\omega)= \begin{cases}1 & \text { for } \omega \in K \cup \overline{\mathbb{D}}_{r}, \\ 0 & \text { for } \omega \notin G .\end{cases}
$$

Then, replacing the path of integration in (3.7) by $\Gamma$ does not change the value of the integral. From the construction of $\Gamma$ it follows that

$$
B:=\left\{\frac{z}{t}\left(\frac{\alpha}{t}\right)^{k}\left(\frac{\beta}{t}\right)^{j q}: z \in C, t \in \Gamma\right\}
$$


is a compact subset of $\Omega$. Setting $M_{\Gamma}:=\max \{|g(t)|: t \in \Gamma\}$, and

$$
\varepsilon_{1}(x):=\max \left\{\left|\phi^{f}(x ; u, w)\right|: u \in B,|w| \leq \frac{1}{r} \max \{|\alpha|,|\beta|\}\right\},
$$

we obtain

$$
\max _{z \in C}\left|T_{k j}(x ; z)\right| \leq \frac{1}{2 \pi r} M_{\Gamma} \operatorname{length}(\Gamma)\left(\frac{|\alpha|}{r}\right)^{k}\left(\frac{|\beta|}{r}\right)^{j q} \varepsilon_{1}(x) \rightarrow 0 \quad\left(x \rightarrow x^{*}\right) .
$$

From (3.8) and (3.9) it follows that

$$
\max _{z \in C}|\psi(x ; z)| \rightarrow 0 \quad\left(x \rightarrow x^{*}\right)
$$

which completes the proof.

Proof of Theorem 2.2. Let $1<r<R$. Then it is well-known that

$$
\begin{aligned}
H_{p(n+1)-1}(z ; g)= & \frac{1}{2 \pi i} \int_{|t|=r} \frac{g(t)}{t-z} \frac{\left(t^{n+1}-1\right)^{p}-\left(z^{n+1}-1\right)^{p}}{\left(t^{n+1}-1\right)^{p}} d t \\
= & \frac{1}{2 \pi i} \int_{|t|=r} \frac{g(t)}{\left(t^{n+1}-1\right)^{p}} \sum_{j=1}^{p}\left(\begin{array}{c}
p \\
j
\end{array}\right)(-1)^{p-j} \\
& \times \sum_{\nu=0}^{j(n+1)-1} t^{j(n+1)-\nu-1} z^{\nu} d t \quad(z \in \mathbb{C}) .
\end{aligned}
$$

Therefore,

$$
\begin{aligned}
& \left(S_{p(n+1)-1}^{f} * H_{p(n+1)-1}(\cdot ; g)\right)(z) \\
& \quad=\frac{1}{2 \pi i} \int_{|t|=r} \frac{g(t)}{\left(t^{n+1}-1\right)^{p}} \sum_{j=1}^{p}\left(\begin{array}{c}
p \\
j
\end{array}\right)(-1)^{p-j} \sum_{\nu=0}^{j(n+1)-1} f_{\nu} t^{j(n+1)-\nu-1} z^{\nu} d t \\
& \quad=\frac{1}{2 \pi i} \int_{|t|=r} \frac{g(t)}{\left(t^{n+1}-1\right)^{p}} \sum_{j=1}^{p}\left(\begin{array}{l}
p \\
j
\end{array}\right)(-1)^{p-j} t^{j(n+1)} S_{j(n+1)-1}^{f}\left(\frac{z}{t}\right) \frac{d t}{t} \quad(z \in \mathbb{C}) .
\end{aligned}
$$

Now we obtain from (3.3) and (3.10)

$$
D_{p n}^{f, g}(z)=\frac{1}{2 \pi i} \int_{|t|=r} g(t) K_{p n}^{f}(z, t) \frac{d t}{t} \quad(z \in \mathbb{C}),
$$

where

$$
\begin{aligned}
K_{p n}^{f}(z, t):= & \frac{1}{\left(t^{n+1}-1\right)^{p}} \sum_{j=1}^{p}\left(\begin{array}{c}
p \\
j
\end{array}\right)(-1)^{p-j} t^{j(n+1)} S_{j(n+1)-1}^{f}\left(\frac{z}{t}\right)-S_{p(n+1)-1}^{f}\left(\frac{z}{t}\right) \\
= & \frac{1}{\left(t^{n+1}-1\right)^{p}} \sum_{j=1}^{p-1}\left(\begin{array}{c}
p \\
j
\end{array}\right)(-1)^{p-j} t^{j(n+1)} S_{j(n+1)-1}^{f}\left(\frac{z}{t}\right) \\
& +\left[\frac{t^{p(n+1)}}{\left(t^{n+1}-1\right)^{p}}-1\right] S_{p(n+1)-1}^{f}\left(\frac{z}{t}\right) .
\end{aligned}
$$


We write

$$
\frac{1}{\left(t^{n+1}-1\right)^{p}}=\sum_{k=0}^{\infty}\left(\begin{array}{c}
p+k-1 \\
k
\end{array}\right) \frac{1}{t^{(k+p)(n+1)}}
$$

and we set

$$
a_{k j}:=\left(\begin{array}{c}
p+k-1 \\
k
\end{array}\right)\left(\begin{array}{l}
p \\
j
\end{array}\right)(-1)^{p-j}
$$

Then we have

$$
\begin{aligned}
K_{p n}^{f}(z, t)= & \sum_{k=0}^{\infty} \sum_{j=1}^{p-1} \sum_{\nu=0}^{j(n+1)-1} a_{k j} \frac{1}{t^{(k+p-j)(n+1)}} f_{\nu}\left(\frac{z}{t}\right)^{\nu} \\
& +\sum_{k=1}^{\infty} \sum_{\nu=0}^{p(n+1)-1} a_{k p} \frac{1}{t^{k(n+1)}} f_{\nu}\left(\frac{z}{t}\right)^{\nu} \\
= & \sum_{\substack{k=0 \\
(k, j) \neq(0, p)}}^{p} \sum_{j=1}^{j(n+1)-1} \sum_{\nu=0}^{a_{k j}} f_{\nu}\left(\frac{z}{t}\right)^{\nu} \frac{1}{t^{(k+p-j)(n+1)}} .
\end{aligned}
$$

For abbreviation, we write $\sum_{k, j}$ instead of $\sum_{\substack{k=0 \\(k, j) \neq(0, p)}}^{\infty} \sum_{\substack{j=1 \\(k)}}^{p}$ the following. Setting

$$
\psi(x ; z, t):=\sum_{n=0}^{\infty} a_{n}(x) K_{p n}^{f}(z, t)
$$

we obtain

$$
\begin{aligned}
\psi(x ; z, t) & =\sum_{k, j} a_{k j} \sum_{n=0}^{\infty} \sum_{\nu=0}^{j(n+1)-1} a_{n}(x) f_{\nu}\left(\frac{z}{t}\right)^{\nu} \frac{1}{t^{(k+p-j)(n+1)}} \\
& =\sum_{k, j} a_{k j} \sum_{\nu=0}^{\infty} \sum_{n=[\nu / j]}^{\infty} a_{n}(x) f_{\nu}\left(\frac{z}{t}\right)^{\nu} \frac{1}{t^{(k+p-j)(n+1)}} \\
& =\sum_{k, j} a_{k j} \sum_{\nu=0}^{\infty} \sum_{n=0}^{\infty} a_{n+[\nu / j]}(x) f_{\nu}\left(\frac{z}{t}\right)^{\nu} \frac{1}{t^{(k+p-j)(n+[\nu / j]+1)}} .
\end{aligned}
$$

Writing $\nu=m j+s$, with $m \in \mathbb{N}_{0}$ and $s \in\{0,1, \ldots, j-1\}$, yields

$$
\begin{aligned}
\psi(x ; z, t) & =\sum_{k, j} a_{k j} \sum_{s=0}^{j-1} \sum_{n=0}^{\infty} \sum_{m=0}^{\infty} a_{n+m}(x) f_{m j+s}\left(\frac{z}{t}\right)^{m j+s} \frac{1}{t^{(k+p-j)(n+m+1)}} \\
& =\sum_{k, j} a_{k j} \frac{1}{t^{k+p-j}} \sum_{n=0}^{\infty} \sum_{m=0}^{\infty} a_{n+m}(x) F_{m j}\left(\frac{z}{t}\right)\left(\frac{z^{j}}{t^{k+p}}\right)^{m} \frac{1}{t^{(k+p-j) n}} \\
& =\sum_{k, j} a_{k j} \frac{1}{t^{k+p-j}} \phi_{j}^{f}\left(x ; \frac{z^{j}}{t^{k+p}}, \frac{1}{t^{k+p-j}}, \frac{z}{t}\right)
\end{aligned}
$$


where $\phi_{j}^{f}(x ; u, w, v)$ and $F_{m j}$ are given by (1.2) and (1.3), respectively. Finally, setting

$$
\psi(x ; z):=\sum_{n=0}^{\infty} a_{n}(x) D_{p n}^{f, g}(z)
$$

and

$$
T_{k j}(x ; z):=\frac{a_{k j}}{2 \pi i} \int_{|t|=r} g(t) \frac{1}{t^{k+p-j}} \phi_{j}^{f}\left(x ; \frac{z^{j}}{t^{k+p}}, \frac{1}{t^{k+p-j}}, \frac{z}{t}\right) \frac{d t}{t},
$$

we see that $\psi(x ; \cdot)$ is an entire function for every $x \in X$, and

$$
\left.\psi(x ; z)=\sum_{\substack{k=0 \\(k, j) \neq(0, p)}}^{\infty} \sum_{\substack{j=1 \\ k j}}^{p} T_{k} ; z\right)
$$

Now the assertion follows by proceeding as in Steps 3 and 4 of the proof of Theorem 2.1 with some simple modifications.

\section{Some remarks on the existence of summability methods}

Finally, we state some results on the existence of summability methods considered in Section 1.2. At first, we remark that most of the classical summability methods for analytic continuation of power series (Euler's methods, Borel's method, Lindelöf's method, etc.) satisfy the condition $(S)$ for certain regions $S$ which contain the unit disk $\mathbb{D}$, and which are star-shaped with respect to 0 . The next two lemmas can be deduced from the proof of the lemma in [3].

Lemma 4.1. If $A$ satisfies the condition $(S)$, and if $\mathbb{D} \subset S$, then $A$ also satisfies the condition $(S, \gamma)$.

Lemma 4.2. If $A$ satisfies the condition $(S)$, if $\mathbb{D} \subset S$, and if $f \in H\left(\Omega^{\prime}\right)$ for some open set $\Omega^{\prime} \subset \mathbb{C}$ such that $0 \in \Omega^{\prime}$, then $A$ also satisfies the condition $\left(\Omega^{\prime} * S, f\right)$.

We remark that $\Omega^{\prime} * S=S$, if $\Omega^{\prime}=\mathbb{C} \backslash\{1\}$, or if $\Omega^{\prime}=\mathbb{C} \backslash[1, \infty)$ and $S$ is star-shaped with respect to 0 .

Furthermore, there is no converse of Lemma 4.2, i.e., there exist summability methods $A$ satisfying the condition $(\Omega, f)$ for some $\Omega$ and some $f$ such that $A$ does not satisfy any condition $(S)$. For example, if we set $X:=(0, \infty), x^{*}:=\infty$, and

$$
a_{n}(x):= \begin{cases}x & \text { for } x>0 \text { and } n=0 \\ 0 & \text { otherwise }\end{cases}
$$

then $\phi^{f}(x ; u, w) \equiv 0$ for every power series $f$ with $f(0)=0$, so that $A$ satisfies the condition $(\mathbb{C}, f)$. But $A$ does not satisfy any condition $(S)$, since $\phi(x ; w) \equiv x \rightarrow \infty$ $\left(x \rightarrow x^{*}\right)$.

The last result in this section gives a sufficient condition for a summability method $A$ to satisfy the condition $(\Omega, f, p)$, and it generalizes Lemma 4.2 . 
Lemma 4.3. If $A$ satisfies the condition $(S)$, if $\mathbb{D} \subset S$, and if $f \in H\left(\Omega^{\prime}\right)$ for some open set $\Omega^{\prime} \subset \mathbb{C}$ such that $0 \in \Omega^{\prime}$, then $A$ also satisfies the condition $\left(\Omega_{p}, f, p\right)$ for any $p \in \mathbb{N}$, where

$$
\Omega_{p}:=\bigcap_{j=1}^{p}\left(\Omega_{(j)}^{\prime} * S\right) \quad \text { and } \quad \Omega_{(j)}^{\prime}=\left(\bigcap_{k=0}^{j-1} e^{2 \pi i k / j} \Omega^{\prime}\right)^{j} \quad(j \in \mathbb{N})
$$

Proof. We use the notations (1.1), (1.2), and (1.3). It suffices to prove that for $j \in \mathbb{N}$

$$
\lim _{x \rightarrow x^{*}} \phi_{j}^{f}(x ; u, w, v)=0 \quad \text { compactly for }(u, w, v) \in\left(\Omega_{(j)}^{\prime} * S\right) \times \mathbb{D} \times \mathbb{C}
$$

We set

$$
F_{j}(z):=F_{j}(z ; v):=\sum_{\nu=0}^{\infty} F_{\nu j}(v) z^{\nu} .
$$

These power series have radii of convergence at least $R_{f}$, and

$$
\phi_{j}^{f}(x ; \cdot, w, v)=F_{j} * \phi^{\gamma}(x ; \cdot, w) .
$$

Now we show that $F_{j} \in H\left(\Omega_{(j)}^{\prime}\right)$. We have

$$
F_{j}(z)=\sum_{\nu=0}^{\infty}\left(\sum_{s=0}^{j-1} f_{\nu j+s} v^{s}\right) z^{\nu}=\sum_{s=0}^{j-1} g_{j s}(z) v^{s}
$$

where

$$
g_{j s}(z):=\sum_{\nu=0}^{\infty} f_{\nu j+s} z^{\nu}
$$

Then, for $w \neq 0$, we consider

$$
G_{j s}(w):=g_{j s}\left(w^{j}\right)=\sum_{\nu=0}^{\infty} f_{\nu j+s} w^{\nu j}=w^{-s} \sum_{\nu=0}^{\infty} f_{\nu j+s} w^{\nu j+s} .
$$

Setting

$$
\gamma_{j s}(w):=\sum_{\nu=0}^{\infty} w^{\nu j+s}=w^{s} \sum_{\nu=0}^{\infty} w^{\nu j}=\frac{w^{s}}{1-w^{j}}
$$

we observe that $\gamma_{j s}$ is holomorphic in $\Omega_{j}:=\mathbb{C} \backslash\left\{e^{2 \pi i k / j}: k=0,1, \ldots, j-1\right\}$, and

$$
w^{s} G_{j s}(w)=\left(f * \gamma_{j s}\right)(w) .
$$

By Theorem H, $f * \gamma_{j s}$ is holomorphic in $\Omega^{\prime} * \Omega_{j}=\bigcap_{k=0}^{j-1} e^{2 \pi i k / j} \Omega^{\prime}$, and therefore, $G_{j s} \in$ $H\left(\Omega^{\prime} * \Omega_{j}\right)$ for $s=0,1, \ldots, j-1$. This implies $g_{j s} \in H\left(\Omega_{(j)}^{\prime}\right)$ for $s=0,1, \ldots, j-1$, and thus $F_{j} \in H\left(\Omega_{(j)}^{\prime}\right)$.

Finally, the assertion (4.1) easily follows from (4.2) and Theorem $\mathrm{H}$ which completes the proof. 


\section{References}

1. R. Brück, Generalizations of Walsh's equiconvergence theorem by the application of summability methods, Mitt. Math. Sem. Giessen 195 (1990), 1-84.

2. - Summability of sequences of polynomial interpolants in the roots of unity, Analysis 11 (1991), 27-42.

3. R. Brück and J. Müller, Summability of Hadamard products of Taylor sections with polynomial interpolants, J. Approx. Theory, to appear.

4. A. S. Cavaretta, Jr., A. Sharma, and R. S. Varga, Interpolation in the roots of unity: An extension of a theorem of J. L. Walsh, Resultate Math. 3 (1980), 155-191.

5. Y. Lou, Extensions of a theorem of J. L. Walsh on the overconvergence, Approx. Theory Appl. 2 (1986), 19-32.

6. J. Müller, The Hadamard multiplication theorem and applications in summability theory, Complex Variables Theory Appl. 18 (1992), 155-166.

7. T. J. Rivlin, On Walsh equiconvergence, J. Approx. Theory 36 (1982), 334-345.

8. J. L. Walsh, Interpolation and Approximation by Rational Functions in the Complex Domain, Amer. Math. Soc., Providence, Rhode Island, 5th ed., 1969.

Mathematisches Institut, Justus-Liebig-Universität Giessen, Arndtstrasse 2, D-35392 Giessen, Germany

Fachbereich 4, Mathematik, Universität Trier, D-54286 Trier, Germany

Department of Mathematics, University of Alberta, Edmonton, Alberta, Canada T6G 2G1 